\title{
REPERCUSSÕES FÍSICAS, EMOCIONAIS E SOCIOECONÔMICAS NOS INDIVÍDUOS QUE VIVENCIAM A ESPERA PROLONGADA POR CIRURGIA
}

\author{
Physical, emotional, and socioeconomic repercussions in individuals \\ who experience the prolonged waiting for surgery
Repercusiones físicas, emocionales y socioeconómicas en individuos que experimentan la espera prolongada por la cirugía

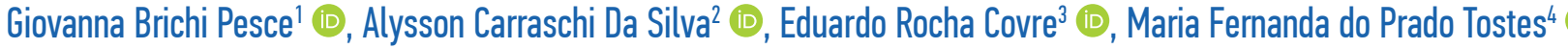

RESUMO: Objetivo: Descrever as repercussões físicas, emocionais e socioeconômicas decorrentes da espera prolongada por cirurgia pelos usuários do Sistema Único de Saúde. Método: Estudo descritivo, com abordagem qualitativa, realizado com base no banco de dados eletrônico "Caixa Preta da Saúde”. Os registros cadastrados foram coletados em 2015. Os resultados foram apresentados em categorias temáticas e interpretados segundo a Teoria de Intervenção Práxica da Enfermagem em Saúde Coletiva. Resultados: A espera prolongada por cirurgia contribuiu para: 1) o agravamento da saúde física, caracterizado principalmente por dores e incapacidades; 2) o sofrimento emocional, constatado pelo desespero, indignação, frustração, entre outros; 3) o impacto socioeconômico relacionado à incapacidade para trabalhar e custear gastos com a saúde. Conclusão: Evidenciou-se que a prolongada espera por cirurgia impactou negativamente a vida dos indivíduos. Acredita-se que a integralidade do cuidado na assistência ao paciente que necessita de cirurgia será garantida se for abordada nas dimensões estrutural, singular e particular.

Palavras-chave: Procedimentos cirúrgicos operatórios. Acesso aos serviços de saúde. Enfermagem.

ABSTRACT: Objective: To describe the physical, emotional, and socioeconomic repercussions resulting from the prolonged waiting for surgery by users of the Brazilian Unified Health System (SUS). Method: Descriptive study with a qualitative approach, carried out based on the electronic database "Caixa Preta da Saúde". The registered records were collected in 2015. The results were presented in thematic categories and interpreted according to the Theory of Praxic Intervention of Nursing in Public Health. Results: The prolonged waiting for surgery contributed to: 1) the worsening of physical health, characterized mainly by pain and disability; 2) emotional suffering, evidenced by despair, indignation, frustration, among others; 3) the socioeconomic impact related to the inability to work and defray health expenses. Conclusion: The prolonged waiting for surgery had a negative impact on the lives of individuals. Comprehensive care in the care of patients who need surgery will be guaranteed if it is addressed in the structural, singular, and particular dimensions.

Keywords: Surgical procedures, operative. Health services accessibility. Nursing.

RESUMEN: Objetivo: Describir las repercusiones físicas, emocionales y socioeconómicas derivadas de la prolongada espera quirúrgica de los usuarios del Sistema Único de Salud. Método: Estudio descriptivo, con abordaje cualitativo, realizado mediante la base de datos electrónica "Caixa Preta da Saúde”. Los registros fueron recolectados en 2015. Los resultados fueron presentados en categorías temáticas e interpretados de acuerdo a la Teoría de la Intervención Práxica de Enfermería en Salud Pública. Resultados: La espera prolongada para la cirugía contribuyó a: 1. Empeoramiento de la salud

\footnotetext{
'Mestre em Enfermagem, Universidade Estadual de Maringá (UEM). Docente colegiado de Enfermagem, Universidade Estadual do Paraná (UNESPAR) - Paranavaí (PR), Brasil. ${ }^{2}$ Acadêmico de Enfermagem, UNESPAR - Paranavaí (PR), Brasil.

${ }^{3}$ Mestre em Enfermagem, UEM. Docente colegiado de Enfermagem, UNESPAR - Paranavaí (PR), Brasil.

¿Doutora em Ciências pela Escola de Enfermagem de Ribeirão Preto, Universidade de São Paulo. Docente colegiado de Enfermagem, UNESPAR - Paranavaí (PR), Brasil.

*Autora correspondente: mfpprado@gmail.com

Recebido: 14/05/2020 - Aprovado: 21/11/2020

https://doi.org/10.5327/Z1414-4425202100010006
} 
física, caracterizada principalmente por dolor y discapacidad; 2. Sufrimiento emocional verificado por desesperación, indignación, frustración, entre otros; 3. Impacto socioeconómico relacionado con la incapacidad para trabajar y los costes sanitarios. Conclusión: Se hizo evidente que la larga espera para la cirugía tuvo un impacto negativo en la vida de las personas. Se cree que la integralidad en la atención de los pacientes que requieren cirugía estará garantizada si se aborda en las dimensiones estructural, singular y particular.

Palabras clave: Procedimientos quirúrgicos operativos. Accesibilidad a los servicios de salud. Enfermería.

\section{INTRODUÇÃO}

A cirurgia é parte indivisível e indispensável dos cuidados de saúde e pode ajudar milhões de pessoas a ter vidas saudáveis e produtivas, portanto deve ser componente fundamental do sistema nacional de saúde em todos os países, independentemente do nível de desenvolvimento socioeconômico ${ }^{1}$.

Nos próximos 20 anos, em decorrência da transição epidemiológica em muitos países de renda baixa e média, a necessidade de cirurgias aumentará contínua e substancialmente ${ }^{1}$. A assistência cirúrgica acessível e segura contribui para a redução da morbimortalidade e de incapacidades decorrentes de condições cirúrgicas. Além disso, melhora o bem-estar da população, a produtividade econômica, a capacidade e liberdade individuais, contribuindo para o desenvolvimento dos países em longo prazo e o fortalecimento dos sistemas de saúde ${ }^{1-4}$.

Apesar da inegável relevância da cirurgia no contexto da saúde pública no Brasil, em pesquisa conduzida em 2014 para avaliar a opinião dos brasileiros sobre o atendimento na área de saúde, a maioria dos respondentes destacou que a cirurgia é um dos procedimentos de acesso mais difícil nos serviços de saúde. Adicionalmente, declarou que a espera é demasiado longa e, para $29 \%$ dos que estão na fila de espera, chega a ser de mais de seis meses 5 . Ademais, a espera pelo tratamento pode ser fator gerador de ansiedade, estresse, incertezas, interferindo nas atividades sociais cotidianas e afetando a capacidade de trabalho ${ }^{6}$.

$\mathrm{Na}$ literatura indexada, este é o primeiro estudo que aborda a repercussão física, emocional e socioeconômica dos usuários do Sistema Único de Saúde (SUS) que se encontram em situação de espera para cirurgia no Sul do Brasil. Assim, diante da importância global da assistência cirúrgica, do seu impacto na qualidade de vida do paciente, da limitação de acesso e do interesse em responder alguns desafios emergentes na Enfermagem, propõe-se o presente estudo.

\section{OBJETIVO}

Descrever as repercussões físicas, emocionais e socioeconômicas decorrentes da espera prolongada por cirurgia pelos usuários do Sistema Único de Saúde.

\section{MÉTODO}

Estudo documental, com abordagem qualitativa, realizado com base em fonte secundária de dados de domínio público do banco de dados eletrônico "Caixa Preta da Saúde". Criado em 12 de março de 2014, fruto de uma iniciativa não governamental da Associação Médica Brasileira (AMB), esse banco é um canal de comunicação eletrônica com os usuários dos serviços de saúde e tem como objetivos receber e compilar denúncias/registros sobre os problemas que afetam a saúde pública e privada no Brasil ${ }^{7}$.

Por meio do endereço eletrônico http:/ / www.caixapretadasaude.org.br/, qualquer pessoa, de qualquer localidade do país, pode se cadastrar e registrar sua denúncia por meio da descrição do evento, selecionando a cidade e o estado.

A coleta de dados foi feita de forma coletiva por dois pesquisadores, em dezembro de 2015. No banco de dados eletrônico, na página eletrônica acima mencionada, uma caixa de seleção estava disponível para selecionar cada estado do país. Após essa escolha, os municípios que possuíam denúncias registradas ficavam disponíveis para seleção. Com a seleção de cada município, abriam-se caixas de texto com o registro da denúncia na íntegra.

No processo de coleta de dados, recuperaram-se os registros do período de um ano a partir da criação da página eletrônica, portanto de março de 2014 a fevereiro de 2015, de cada município da região Sul do país. Os registros recuperados foram separados por estados e armazenados em planilhas Excel. Em cada uma destas, as seguintes variáveis foram preenchidas: data da denúncia, município, serviço de saúde envolvido, descrição completa, relação com 
assistência cirúrgica (sim ou não), relação com o tempo de espera prolongada (sim ou não). Cada registro foi lido na íntegra e foram selecionados aqueles que atendiam ao seguinte critério de inclusão: a denúncia deveria abordar a espera para a cirurgia e as repercussões decorrentes dessa espera na vida do indivíduo.

$\mathrm{Na}$ análise dos dados, outros dois pesquisadores participaram coletivamente do processo. Os relatos foram agrupados em categorias temáticas e analisados qualitativamente. Para a codificação dos relatos, a cidade de origem da denúncia e o tempo de espera para a cirurgia foram mencionados.

Neste estudo, elegeu-se como base teórica a Teoria de Intervenção Práxica da Enfermagem em Saúde Coletiva (TIPESC). Assentada na visão de mundo materialista, histórica e dialética, essa teoria é portanto nascida no campo da saúde coletiva e no marco da determinação social dos processos de saúde-doença. Busca captar e interpretar um fenômeno articulado aos processos de produção e reprodução social referentes à saúde-doença de uma dada coletividade, no marco de sua conjuntura e estrutura, em um contexto social historicamente determinado: o fenômeno de intervir nessa realidade e prosseguir reinterpretando a realidade objetiva para novamente interpor um instrumento de intervenção.

Existem três dimensões da realidade objetiva na operacionalização da TIPESC: a dimensão estrutural, a particular e a singular. A estrutural é a aproximação dos aspectos macroscópicos ou macroestruturais do objeto focalizado. A particular refere-se ao perfil epidemiológico de classe, ao perfil reprodutivo, ao perfil saúde-doença e a práticas e ideologias em saúde. A singular destaca os processos que levam ao adoecer-morrer ou ao desenvolvimento do nexo biopsíquico dado pelo funcionamento e consumo-trabalho individual do homem ${ }^{8}$.
O estudo obedeceu à Resolução n ${ }^{\circ}$ 466/ 2012 do Conselho Nacional de Saúde, e o projeto foi aprovado pelo Comitê de Ética, via Plataforma Brasil, sob Certificado de Apresentação para Apreciação Ética (CAAE) n ${ }^{\circ} 44899215.0 .0000 .0104$ e parecer $1.124 .424 / 2015$

\section{RESULTADOS}

Segundo dados da Caixa Preta da Saúde, 3.773 denúncias que envolviam assistência à saúde estavam registradas e eram provenientes de todas as regiões do Brasil. Na região Sul, havia 462 denúncias, das quais 72 envolviam assistência cirúrgica. Destas, a maioria $(\mathrm{n}=51)$ mencionava dificuldades de acesso à cirurgia. Além disso, houve predomínio de denúncias $(\mathrm{n}=26)$ que relatavam repercussão física, seguida das que abordavam a repercussão emocional $(n=17)$ e de oito que mencionavam o impacto socioeconômico. Na maioria dos relatos, os pacientes foram acometidos simultaneamente pelas repercussões físicas, emocionais e/ ou socioeconômicas decorrentes da situação de espera para cirurgia, conforme Tabela 1.

A seguir, os relatos que envolviam a espera prolongada pela assistência cirúrgica são categorizados e apresentados em três categorias.

\section{Agravamento da saúde física decorrente da prolongada espera por cirurgia}

Em relação aos relatos, constatou-se que o prolongado tempo de espera contribuiu para o surgimento de desconforto físico evidenciado por dor, piora da dor ou limitações físicas do paciente, impedindo-o de realizar atividades rotineiras que antes eram consideradas simples. Houve também outros sintomas relatados, conforme os trechos a seguir:

Tabela 1. Caracterização das denúncias registradas na Caixa Preta da Saúde, diferenciadas por estados da Região Sul do Brasil.

\begin{tabular}{|l|c|c|c|c|}
\hline \multirow{2}{*}{ Variáveis } & \multicolumn{4}{|c|}{ Estados da Região Sul } \\
\cline { 2 - 5 } & Paraná & Santa Catarina & Rio Grande do Sul & Total \\
\hline Tema das denúncias & & & 188 & 462 \\
\hline Geral & 185 & 89 & 21 & 72 \\
\hline Assistência cirúrgica & 35 & 16 & 16 & 51 \\
\hline Espera por cirurgia & 26 & 9 & 8 & 26 \\
\hline Repercussões decorrentes da espera prolongada por cirurgia & 14 & 4 & 6 & 17 \\
\hline Físicas & 7 & 4 & 2 & 8 \\
\hline Emocionais & 5 & 1 & \\
\hline Socioeconômicas & 5 & & 2 \\
\hline
\end{tabular}


"Liberação de cirurgia da vesícula biliar [...] só para 29 de setembro... sofro dores constantes" (Cachoeira do Sul/RS, cinco meses de espera).

"Estou desde 2010 na fila para cirurgia de amígdalas [...], estou tendo dores na nuca passando pelo pescoço indo no ouvido" (Londrina/PR, cinco anos de espera).

"Ela teve uma torção no fêmur que quando foi ver tinha quebrado, mas como estava fraca dos ossos, os médicos alegaram que não poderia ser feita a cirurgia, então ela já está assim há quatro anos em cima de uma cadeira de rodas" (Porto Alegre/RS, quatro anos de espera).

"Desde 2008 espero uma cirurgia de retirada de materiais no cotovelo [...], sinto muitas dores" (Joinville/ $\mathrm{SC}$, seis anos de espera).

"Meu pai está internado na UPA [...], mas até hoje ele não conseguiu uma vaga para cirurgião vascular. Ele tem febre todos os dias" (Curitiba/PR, tempo de espera não mencionado).

"Meu sogro [...] apresenta entupimento de $90 \%$ da carótida, comprometendo a vascularização do cérebro, tendo que efetuar cirurgia mais que urgente [...], até hoje não foi feita a cirurgia, tem tonturas e perda de concatenação de ideias" (Maringá/PR, oito meses de espera).

"Minha irmã $[\ldots]$ está esperando pela cirurgia bariátrica há mais de três anos, ficou cega pelas complicações do diabetes" (Canoas/RS, três anos de espera).

Apesar de o tempo de espera por atendimento não ter sido longo, colocou o paciente em risco. Adicionalmente, a espera por cirurgia deu-se em razão da falta de materiais, conforme descrições a seguir:

"Gestante há três dias com bebê morto na barriga ainda aguarda cesárea" (Rolândia/PR, três dias).

"Estou com um cálculo renal de $0,8 \mathrm{~cm}$ parado no ureter, preciso fazer a cirurgia de colocação de cateter duplo $J$ [...], eu teria que aguardar porque não tem materiais para a cirurgia, tenho cólicas constantes [...], corro o risco de perder a função do rim esquerdo" (Londrina / PR, 20 dias de espera).

\section{Sofrimento emocional de pacientes e familiares decorrentes da prolongada espera por cirurgia}

Nesta categoria foram identificados sentimentos como desespero, impotência, sofrimento psíquico, frustração, vergonha, sentimento de desrespeito, humilhação, descaso, descrença, revolta contra o SUS e o atendimento realizado por alguns profissionais. Pacientes e familiares vivenciaram preconceito e constrangimento:

"Minha tia foi diagnosticada com um tumor no osso da perna e já faz mais um de ano [...], gostaria de pedir ajuda pelo amor de Deus" (São João do Sul/SC, mais de um ano de espera).

"Estou aguardando uma cirurgia há sete anos [...], não sei mais o que fazer" (Curitiba/PR, sete anos de espera).

"Tenho hemorroidas [...], passados quase dois anos, me ligaram marcando a cirurgia [...], depois ligaram desmarcando devido ao feriado [...]. Achei um desrespeito comigo, me senti humilhada [...], vim para casa frustrada, me sentindo envergonhada de ser brasileira, trabalhadora" (Florianópolis/SC, proctologia, dois anos de espera).

"Fui encaminhada para a colocação de prótese no joetho há quase oito anos e até agora nada [...], descaso com o povo brasileiro!" (Guaíba/RS, ortopedia, oito anos de espera).

"Estou aguardando cirurgia vascular de membros inferiores há cinco anos [...], estou com medo de perder minhas pernas. Deus me ajude! Paguei [Instituto Nacional do Seguro Social] INSS a vida inteira e agora que preciso me viram as costas" (Porto Alegre/ RS, vascular, cinco anos de espera).

"Já faz quatro anos que estou na espera cirurgia bariátrica [...], sem contar o preconceito que todos os obesos enfrentam no dia a dia" (Sapiranga/RS, quatro anos de espera). 
Verificou-se que, em alguns casos, o período de espera evoluiu para condições mais graves, como o óbito. Nessas situações, as repercussões não ficaram restritas ao indivíduo, mas abarcaram pessoas próximas e familiares. Além disso, pelos relatos, verificam-se situações em que houve negligência no atendimento, conforme relatos a seguir:

"Perdi uma amiga, simplesmente por médicos não tocarem em pacientes ao atendê-los; o apêndice dela estourou [...], teve que esperar 13 horas por uma cirurgia que deveria ter sido feita com urgência [...], perdeu a vida por irresponsabilidade e demora no atendimento" (Rio Grande/RS, 13 horas).

\begin{abstract}
"Meu pai [...], 89 anos, estava aguardando [...] uma consulta com médico cardiologista, porque necessitava de um marca-passo [...], infelizmente seu coração não aguentou a espera" (Viamão/RS, cinco meses).
\end{abstract}

"Meu pai precisou ser atendido no pronto-socorro [...] pois estava com fortes dores no abdômen [...], aplicaram dolantina, um potente remédio para dor... Houve uma melhora no quadro da dor e meu pai foi liberado [...]. Quando foi meia-noite (já sábado) meu pai entra no PA novamente com a mesma queixa [...], meu pai ficou sem assistência de um profissional até as 16 horas [...]. Minha mãe estava desesperada [...]. Quando abriram era uma úlcera perfurada, o intestino havia necrosado, um rim não funcionava mais... Enfim... Meu pai faleceu" (Guaíba/RS, 16 horas).

\section{Impacto socioeconômico decorrente da prolongada espera por cirurgia}

Nesta categoria, a impossibilidade de trabalhar oriunda do agravamento da saúde física foi uma queixa frequente, segundo as narrações que se seguem:

"Estou à espera de uma cirurgia de varizes desde de agosto de 2012 [...], estou sem trabalhar, pois sinto muita dor" (Santa Maria/RS, três anos de espera).

"Rompi o ligamento cruzado do meu joelho esquerdo há três anos [...], não estou conseguindo trabalhar" (Almirante Tamandaré/PR, público, três anos de espera).
"Eu aguardo a cirurgia até a data de hoje [...], não posso mais trabalhar devido à hérnia" (Curitiba/PR, um ano e seis meses de espera).

"Minha mãe está na fila para a cirurgia de redução do estômago há três anos [...], ela não consegue mais trabalhar, e nada da cirurgia" (Londrina/PR, três anos de espera).

"Meu marido está aguardando uma cirurgia do joelho [...]. Ele está afastado do trabalho por tempo indeterminado" (Londrina/PR, tempo de espera não mencionado).

"Para fazer cirurgia de tireoide, que está em estado avançado e penetrando na caixa torácica, tinha 2.287 pessoas na fila [...], vamos ter que vender coisas (carro, outros objetos) para fazer a cirurgia particular" (Foz do Iguaçu/PR, tempo de espera não mencionado).

\section{DISCUSSÃO}

Este estudo revelou que a prolongada espera por cirurgia vivenciada pelos usuários do SUS na região Sul do Brasil repercutiu de forma abrangente e negativa na vida dos indivíduos. Destaca-se que, além do agravamento da condição física, problemas de ordem emocional e social emergiram dessa inaceitável condição de espera.

Em estudo conduzido com propósito similar, os autores mostraram que a espera pela cirurgia envolveu um período prolongado de saúde diminuída na vida dos indivíduos. Além da condição física, as dimensões psicológica e social foram afetadas. A variação na gravidade dessas consequências entre os pacientes indica que a priorização de casos poderia reduzir essa carga pessoal decorrente da espera. Informações precoces sobre a duração do atraso podem promover ainda mais a aceitação do paciente pela espera ${ }^{6}$.

Na tentativa de lançar um olhar mais amplo sobre essa problemática e suas contradições, faz-se necessário compreendê-la e abordá-la dos pontos de vista estrutural, particular e individual, pois se acredita que esse fenômeno decorre das contradições encontradas na interpretação dessa dramática realidade.

Em análise do ponto de vista estrutural, recorre-se à Constituição Federal de 1988, que põe em pauta a saúde como direito de todos e dever do Estado, garantida mediante políticas sociais e econômicas para a redução do risco de doença 
e outros agravos e acesso universal e igualitário às ações e serviços para sua promoção, proteção e recuperação. Para a operacionalização da política de saúde, criou-se o SUS, com o propósito de oferecer à população brasileira serviços de saúde desde a atenção primária à terciária, pautados nos seguintes princípios: universalidade, equidade e integralidade ${ }^{9}$.

Diante da falta de acesso e de garantia da assistência cirúrgica, evidenciada pelos resultados deste estudo, observa-se que a política de saúde vigente, pautada por princípios sólidos, não cumpriu seu propósito. Acredita-se que um dos fatores contribuintes é o histórico e inaceitável subfinanciamento, além da gestão ineficiente do setor saúde no Brasil, com gasto público per capita em saúde inferior ao de países com sistemas universais similares ao nosso ${ }^{7}$.

Entre os serviços fornecidos pelo SUS, o procedimento cirúrgico é o segundo mais requisitado. Como consequência, em 2015, foram destinados 143,2 milhões de reais para as cirurgias eletivas, conforme regulamentação pela portaria 1.034 do Ministério da Saúde ${ }^{10}$. Entretanto, observa-se que iniciativas governamentais como essa são importantes, mas não resolutivas para quem vivencia a situação de espera. Para superar esse desafio macroestrutural, faz-se necessário vontade política para que a saúde seja prioridade governamental.

A demora pelo atendimento levou alguns usuários a declararem ter procurado o serviço particular de saúde. Assim, os mecanismos de instalação de uma medicina privada como sistema principal e não suplementar robustecem-se, fortalecendo a crise na saúde pública brasileira. Crise esta iniciada no pós-Constituição Federal de 1988 como um contrassenso normativo, pois, se de um lado um sistema legal promete uma saúde universal e irrestrita, de outro, a realidade de pouca concretude do direito de cidadania à saúde no Brasil fica evidente no seu subfinanciamento ${ }^{11}$.

Uma vez que se estima que 5 bilhões de pessoas não tenham acesso aos cuidados anestésico e cirúrgico quando necessários, em 2013 foi criada a Lancet Commission on Global Surgery para avaliar o estado atual do atendimento cirúrgico mundial e fazer recomendações concretas em prol do acesso universal aos cuidados anestésicos e cirúrgicos. Essa comissão estabeleceu a realização de 5 mil procedimentos cirúrgicos anuais por 100 mil pessoas até 2030. Esse indicador corresponde a um suprimento adequado das necessidades em cuidados anestésicos e cirúrgicos. Para atingir esse objetivo, será imperativo assegurar uma larga expansão dos sistemas de saúde e cirúrgicos, o que implica contratar o dobro da força laboral cirúrgica até 2030. Essa expansão do volume cirúrgico deverá ser acompanhada por um reforço de qualidade, segurança e equidade, que deve ser garantido pelos gestores locais nos países ${ }^{12}$.

Na dimensão particular, revelam-se muitas falhas de acesso decorrentes da má gestão dos serviços de saúde. As causas organizacionais relativas às instituições de saúde são: falta de leitos e de profissionais, erros de agendamento e falhas de comunicação, bem como problemas administrativos de outra ordem $^{13}$. Como exemplo disso, em estudo conduzido para investigar o número de cirurgias eletivas canceladas em um hospital universitário e identificar as suas causas, os autores apontaram que, durante três meses, 1.699 cirurgias eletivas foram agendadas, das quais $466(27,4 \%)$ foram canceladas. A maioria dos cancelamentos deu-se "a critério do cirurgião", o que foi constatado em 264 casos (56,7\%), sem que se informasse especificamente o motivo da decisão ${ }^{14}$.

Durante o período anterior à cirurgia, pode-se pensar que os indivíduos tenham permanecido sem qualquer acompanhamento pelos serviços e profissionais da área da saúde, o que foi demonstrado nos relatos caracterizados por insegurança, temor, revolta e descrenças em relação a esses serviços. Essa desassistência e completa ausência de vínculo entre indivíduos e profissionais de saúde fere amplamente o princípio da integralidade. Essa realidade não é exclusiva do Brasil, pois em estudo realizado no Canadá se observou que os pacientes em espera por cirurgia bariátrica não receberam assistência no período anterior ao procedimento, sendo sugerido pelos pesquisadores que o sistema de saúde deveria fornecer uma pessoa para contato que pudesse dar informações sobre o tempo de espera, o lugar na lista de espera e explicações sobre os atrasos ${ }^{15}$.

Essa lacuna da assistência ao paciente em fase de espera, para aquele que necessita de cirurgia, remete-nos à atenção básica, já que ela é a porta de entrada do usuário ao SUS para posterior encaminhamento ao serviço especializado $^{16}$. Neste estudo, os resultados revelaram a existência de demandas de cuidado aos indivíduos, seja para minimizar agravos decorrentes do processo patológico, seja para prevenir a ocorrência de outras desordens psicossociais oriundas da situação de espera. Sugere-se assim que, no âmbito da atenção básica e especializada, tem havido negligência no cuidar. Nesse aspecto, acredita-se que a Enfermagem, no cuidado ao paciente com necessidade cirúrgica, possa atuar de forma relevante extramuros ao Centro Cirúrgico/Hospital. Isso porque o enfermeiro, na atenção primária e secundária, tem papel fundamental nesse processo e pode exercer um protagonismo nas 
ações de cuidado preventivo aos agravos oriundos da espera por cirurgia e, com isso, fortalecer a assistência à saúde daqueles que esperam pelo procedimento.

$\mathrm{Na}$ Inglaterra, estratégia considerada bem-sucedida para gerenciar a espera é a definição de tempo mínimo recomendado e estabelecido para diferentes tipos de procedimentos eletivos e a associação de incentivos hospitalares ou penalidades ao cumprimento desse objetivo. Ainda como intervenções para reduzir a longa lista de espera, podem-se realizar ações de triagem, ou seja, priorizar casos levando em consideração a gravidade da doença, o benefício que o procedimento cirúrgico traria e a possibilidade ou não de se trabalhar de forma clínica com esse paciente. ${ }^{17}$

No âmbito singular, notou-se que esses cidadãos se encontram em um estado de vulnerabilidade, fragilizados na garantia dos seus direitos de cidadãos. A espera prolongada pode evoluir para piora do processo saúde-doença, resultante do espaço de produção social, caracterizado por iniquidades e desassistência ${ }^{18}$. Ainda, a condição física e emocional prejudicada impedia que as funções profissionais fossem exercidas, funções estas que não são apenas fonte de sustento, mas também de status e parte da identidade pessoal.

Diante disso, é notória a determinação social da saúde no dilema da espera por cirurgia, fruto das seguintes contradições sociais: falta de acesso à cirurgia e desassistência nos demais níveis de atenção para prevenir agravos físicos e psicossociais decorrentes da espera prolongada por cirurgia. Isso revela a invisibilidade das ações assistenciais, até mesmo do enfermeiro, nesse campo que é profícuo para sua atuação e para o exercício do protagonismo da Enfermagem.

Como limitação, sublinha-se que este estudo abarcou os dilemas oriundos dos pacientes que vivenciaram a espera prolongada por cirurgia no Sul do Brasil e que foram registrados voluntariamente no banco de dados consultado, portanto, essa realidade pode não ser representativa no país.

Apesar disso, acredita-se que esta investigação oportunizou conhecer a situação dos usuários dos serviços de saúde em espera para cirurgia, nas três dimensões da realidade objetiva. Na dimensão estrutural, analisou-se a questão na perspectiva do papel governamental do Estado e suas contradições: saúde como garantia constitucional e política de saúde com fundamentos e princípios sólidos, mas que não é amplamente garantida aos cidadãos. Por não ser prioridade de governo, o Estado deserta do seu papel de garanti-la. Na dimensão particular, a reorganização dos serviços de saúde deve oportunizar a assistência à saúde integral em todos os níveis de atenção, entretanto a contradição é evidenciada na gestão ineficiente e na desassistência promovida pelos serviços e profissionais de saúde. Na dimensão singular, destaca-se a seguinte contradição: os indiví duos apresentam necessidades de saúde que ultrapassam o escopo do processo patológico que o gerou, por estarem desassistidos, e outros problemas e demandas por cuidado surgem nos aspectos físico, mental e social.

Em relação às implicações para a Enfermagem, estes resultados atuam como um chamamento aos enfermeiros de todos os níveis de atenção à saúde, uma vez que o paciente cirúrgico se encontra nos diferentes pontos da rede de atenção e, logo, deve ser cuidado em sua integralidade para o fortalecimento do sistema de saúde e da política de saúde vigente.

\section{CONSIDERAÇÕES FINAIS}

As principais repercussões decorrentes da espera por cirurgia vivenciada pelos indivíduos abarcaram, primeiramente, os aspectos físicos, caracterizados principalmente por dores e incapacidade física e outras alterações fisiológicas. Constataram-se as seguintes repercussões emocionais: desespero, indignação, frustação, negligência, preconceito e constrangimento. Nas implicações de ordem socioeconômica, destacaram-se: incapacidade de trabalhar e custeio de gastos excessivos com o setor particular de saúde. Os pacientes que recorreram ao endereço eletrônico da Caixa Preta da Saúde o fizeram em tom de desabafo, mencionando somente os aspectos negativos que a espera por um procedimento cirúrgico proporcionou em suas vidas, independentemente da natureza desses impactos.

Ressalta-se que os depoimentos carregam amplo descontentamento e insatisfação com a atual situação do SUS no Brasil. Não houve relatos que revelassem experiências positivas com o serviço ou a atuação de profissionais durante o período de espera. Ao contar sua história pessoal ou de seu familiar ou amigo, o indivíduo teve a oportunidade de expor seus temores e a preocupação com a saúde, na expectativa de que o agendamento da cirurgia fosse agilizado. Isso mostra que esse infortúnio individual, em sua essência, é coletivo, envolvendo familiares, amigos, serviços, profissionais de saúde e o Estado.

Em conclusão, a prolongada espera por cirurgia é um problema multifatorial. Acredita-se que a integralidade do cuidado na assistência ao paciente que necessita de cirurgia só poderá ser garantida quando for compreendida e abordada em todas as suas dimensões. 


\section{REFERÊNCIAS}

1. Meara JG, Leather AJM, Hagander L, Alkire BC, Alonso N, Ameh EA, et al. Global Surgery 2030: evidence and solutions for achieving health, welfare, and economic development. Lancet. 2015;386(9993):569-624. https://doi.org/10.1016/ s0140-6736(15)60160-x

2. Weiser TG, Haynes AB, Molina G, Lipsitz SR, Esquivel MM, UribeLeitz T, et al. Size and distribution of the global volume of surgery in 2012. Bull World Health Organ. 2016;94(3):201-9F. https://doi. org/10.2471\%2FBLT.15.159293

3. Organização Mundial da Saúde. Segundo desafio global para a segurança do paciente: Cirurgias seguras salvam vidas. Rio de Janeiro: Organização Pan-Americana da Saúde; 2009.

4. Mock CN, Donkor P, Gawande A, Jamilson DT, Kruk ME, Debas HT. Essential surgery: key messages from disease control priorities. Lancet. 2015;385(9983):2209-19. https://doi.org/10.1016/ s0140-6736(15)60091-5

5. Conselho Federal de Medicina. Opinião dos brasileiros sobre o atendimento na área de saúde. Datafolha. 2014.

6. Oudhoff JP, Timmermans DRM, Knol DL, Bijnen AB, Van Der Wal $G$. Waiting for elective general surgery: impact on health-related quality of life and psychosocial consequences. BMC Public Health. 2007;7:164-74. https://doi.org/10.1186/1471-2458-7-164

7. Tostes MFP, Covre ER, Fernandes CAM. Access to surgical assistance: challenges and perspectives. Rev Latino-Am Enferm. 2016;24:e2677. https://doi.org/10.1590/1518-8345.0954.2677

8. Egry EY. Saúde coletiva: construindo um novo modelo em enfermagem. São Paulo: Ícone; 1996.

9. Brasil. Lei Orgânica de Saúde nº 8.080, de 19 de setembro de 1990. Dispõe sobre as condições para a promoção, proteção e recuperação de saúde, a organização e o funcionamento dos serviços correspondentes e dá outras providências. Diário Oficial da União. 1990.
10. Brasil. Ministério daSaúde. Portarian ${ }^{\circ} 1.034$, de 22 de julho de 2015. Redefine aestratégia para ampliação do acesso aos procedimentos cirúrgicos eletivos de média complexidade, no âmbito do Sistema Único de Saúde (SUS) para o exercício de 2015. Brasília: Ministério da Saúde; 2015.

11. Paiva WS, Lima AJ. A financeirização da saúde pública no Brasil: uma análise do subfinanciamento da área da saúde e da priorização do campo privado na saúde brasileira. Rev FSA. 2014;11(2):350-65. http://doi.org/10.12819/2014.11.2.19

12. Uribe-Leitz T, Esquivel MM, Molina G, Lipsitz SR, Verguet S, Rose J, et al. Projections for Achieving the Lancet Commission Recommended Surgical Rate of 5000 operations per 100,000 population by regionspecific surgical rate estimates. World J Surg. 2015;39(9):2168-72. https://doi.org/10.1007/s00268-015-3113-6

13. AvilaAG, BocchiSCM. Telephoneconfirmation of a patient's intent to be present for elective surgery as a strategy to reduce absenteeism. Rev Esc Enferm USP. 2013;47(1):93-7.https://doi.org/10.1590/S0080-62342013000100024

14. Botazini NO, Toledo LD, Souza DMST. Cirurgias eletivas: cancelamentos e causas. Rev SOBECC. 2015;20(4):210-9. https://doi.org/10.5327/ Z1414-4425201500040005

15. Gregory DM, Newhook JT, Twells L. Patient's perceptions of waiting for bariatric surgery: a qualitative study. Int J Equity Health. 2013;12:8697. https://doi.org/10.1186/1475-9276-12-86

16. Paim J, Travassos C, Almeida C, Bahia L, Macinko J. The Brazilian health system: history, advances, and challenges. Lancet. 2011;37(9779):177897. https://doi.org/10.1016/S0140-6736(11)60054-8

17. Ballini L, NegroA, MaltoniS, VignatelliL, Flodgren G, Simeral, etal. Interventions to reduce waiting times for elective procedures. Cochrane Database Syst Rev. 2015;(2):CD005610. https://doi.org/10.1002/14651858.cd005610.pub2

18. Pareja JMD, Guerra FF, Vieira SR, Teixeira KMD. A produção do espaço e sua relação no processo de saúde-doença familiar. Saúde Soc. 2016;25(1):133-44. https://doi.org/10.1590/S0104-12902016152797 